\title{
HIDROGENAÇÃo DE COMPOSTOS ORGÂNICOS UTILIZANDO MÉTODO ELETROQUÍMICO PARA GERAÇÃO DE HIDROGÊNIO in situ: HIDROGENAÇÃO ELETROCATALÍTICA
}

Daniela Maria do Amaral Ferraz Navarro e Marcelo Navarro*

Departamento de Química Fundamental, Centro de Ciências Exatas e Naturais, Universidade Federal de Pernambuco, 50670-901 Recife - PE

Recebido em 27/1/03; aceito em 8/10/03

\begin{abstract}
HYDROGENATION OF ORGANIC COMPOUNDS BY AN ELECTROCHEMICAL METHOD FOR IN SITU HYDROGEN GENERATION: ELECTROCATALYTIC HYDROGENATION. Electrocatalytic hydrogenation (HEC) may be compared to catalytic hydrogenation (HC). The difference between these methods is the hydrogen source: HC needs a hydrogen gas supply; HEC needs a source of protons (solvent) to be reduced at a cathode surface. HEC has presented interesting advances in the last decades due to investigation of the influence of the supporting electrolyte, co-solvent, surfactant, presence of inert gas and the composition of the electrode on the reaction. Several classes of organic compounds have been hydrogenated through HEC: olefins, ketones, aldehydes, aromatics, polyaromatics and nitro-compounds. This paper shows some details about the HEC which may be regarded as a promising technique for the hydrogenation of organic compounds both in industrial processes and in laboratories.
\end{abstract}

Keywords: electrocatalytic hydrogenation; reduction of organic compounds; electrocatalysis.

\section{INTRODUÇÃO}

Dentre as várias reações disponíveis para redução de compostos orgânicos, a hidrogenação catalítica (HC) - a reação de um composto com hidrogênio na presença de um catalisador - oferece vantagens de larga aplicabilidade e simplicidade experimental única ${ }^{1}$. A HC é usualmente efetuada no laboratório pela agitação da solução do composto a ser reduzido, com um catalisador heterogêneo e sob atmosfera de gás hidrogênio. $O$ processo de redução pode ser seguido pela medida de consumo de hidrogênio, e o produto bruto de redução é usualmente isolado pela simples filtração do catalisador, seguido pela evaporação do solvente. Podemos citar também a hidrogenação catalítica homogênea, devido à sua importância na síntese enantiosseletiva. Neste caso, são usados complexos de metais de transição coordenados a ligantes contendo um centro estereogênico capaz de realizar o reconhecimento quiral do reagente para originar produtos com excesso enantiomérico ${ }^{2}$.

A HC de compostos orgânicos é uma das reações mais estudadas da catálise heterogênea (mais de 1000 citações na literatura) ${ }^{1}$. Sua utilização ocorre tanto em pequena como em grande escala. Podemos verificar a introdução da $\mathrm{HC}$ em uma ou mais etapas de diversos processos industriais em áreas como petroquímica, alimentícia e farmacêutica.

A geração de hidrogênio via eletroquímica promove um outro método para hidrogenação de compostos orgânicos ${ }^{3}$. A hidrogenação eletrocatalítica (HEC), como é chamada, é conhecida desde o início do século passado ${ }^{4}$, quando foi sugerido que reduções eletroquímicas realizadas em cátodos de metais de transição deveriam envolver reações entre substratos insaturados e átomos de hidrogênio adsorvidos na superfície do eletrodo gerados pela descarga eletrônica de prótons. Entretanto, a HEC não teve o mesmo sucesso da $\mathrm{HC}$, provavelmente devido à necessidade de um aparato eletroquímico, condições reacionais e a fraca reatividade dos materiais eletródicos adicionada à baixa densidade de corrente necessária para a eficiência dos resultados ${ }^{5}$.

*e-mail: navarro@ufpe.br
Na última década ocorreu uma mudança do ponto de vista com relação ao processo eletroquímico e suas vantagens, ocorrendo o desenvolvimento de eletrodos diferenciados capazes de hidrogenar seletivamente diferentes classes de substratos orgânicos ${ }^{3}$. Outro importante avanço foi a descoberta da influência de diferentes parâmetros pertinentes ao processo eletroquímico, como eletrólito de suporte $^{6}$, solvente ${ }^{7}$ e presença de surfactantes ${ }^{8}$ ou gás inerte 9 .

O sucesso da HEC está associado à conjugação de dois mecanismos: o de geração de hidrogênio ${ }^{10}$ e o de hidrogenação catalítica ${ }^{1}$. O primeiro, também chamado de reação de evolução de hidrogênio $(\mathrm{REH})^{11-13}$, está classicamente baseado na etapa de descarga elétrica primária gerando o hidrogênio atômico, que permanece na superfície do metal por adsorção química:

$\mathrm{H}^{+}{ }_{(\mathrm{aq})}+\mathrm{e}-\longrightarrow \mathrm{H}_{(\mathrm{ad})}^{\cdot}$

(1) Volmer

A etapa seguinte pode ser tanto a reação de recombinação de $\mathrm{H}^{*}$ :

$\mathrm{H}_{(\mathrm{ad})}^{\cdot}+\mathrm{H}_{(\mathrm{ad})}^{\cdot} \longrightarrow \mathrm{H}_{2}$

(2) Tafel

Como a reação entre um próton, átomo e elétron (desorção eletroquímica):

$\mathrm{H}_{(\text {ad) }}^{\cdot}+\mathrm{H}_{(\text {aq) }}^{+}+\mathrm{e}-\longrightarrow \mathrm{H}_{2}$

(3) Heyrovsky

No caso de as etapas serem estritamente consecutivas e uma delas ser a etapa determinante da reação (e.d.r.), a teoria ${ }^{14}$ prevê um coeficiente angular de Tafel de $120 \mathrm{mV}$ para a reação (1), também chamada de etapa de Volmer; de $30 \mathrm{mV}$ para a reação (2), etapa de Tafel; e de $40 \mathrm{mV}$ para a reação (3), etapa de Heyrovsky. Portanto, após determinação do coeficiente angular de Tafel pode-se prever a e.d.r. nas condições reacionais para a HEC. A variação na e.d.r. depende do intervalo de potencial de trabalho, portanto é importante identificar qual o intervalo de potencial para a reação de redução do próton na superfície catalítica, para evitar perdas através da REH, levando a uma baixa eficiência eletroquímica ${ }^{15}$. 
O processo de HEC estará completo após outras três etapas ${ }^{1}$ características para a reação de $\mathrm{HC}$, em outras palavras adsorção do substrato na superfície do metal (4), hidrogenação do substrato (5) e dessorção do produto hidrogenado da superfície do eletrodo (6):

$\mathrm{Y}=\mathrm{Z} \stackrel{\mathrm{M}}{\longrightarrow}(\mathrm{Y}=\mathrm{Z})_{(\text {(ad.) }}$

(4) adsorção

$(\mathrm{Y}=\mathrm{Z})_{(\text {ad.) }}+2 \mathrm{H}_{(\text {ad.) }} \stackrel{\mathrm{M}}{\longrightarrow}(\mathrm{YH}-\mathrm{ZH})_{(\text {ad. })}$

(5) hidrogenação

$(\mathrm{YH}-\mathrm{ZH})_{(\text {ad. })} \stackrel{\mathrm{M}}{\longrightarrow}(\mathrm{YH}-\mathrm{ZH})$

(6) dessorção

Todo este processo depende do material do cátodo (M), uma vez que o mesmo possui dupla função, como gerador de hidrogênio atômico e catalisador.

Como o processo de geração de hidrogênio é eletroquímico, moléculas eletroativas (baixo potencial de redução) ${ }^{16}$ contendo funções orgânicas como duplas ligações conjugadas, aldeídos e nitrocompostos, podem sofrer a transferência direta de um ou mais elétrons no mesmo potencial de redução da água. Este processo pode proporcionar uma competição entre a HEC e reações eletroquímicas diretas, que geram radical-ânions que podem formar dímeros (dimerização eletroquímica - DE) ${ }^{17}$, hidrogenação de reagentes (hidrogenação eletroquímica - HE), além de outros produtos. A hidrogenação eletroquímica e dimerização, freqüentemente observadas num mesmo sistema, são reações concorrentes em que o produto reacional pode ser controlado pela escolha de condições experimentais ideais (Esquema 1) ${ }^{18-20}$.

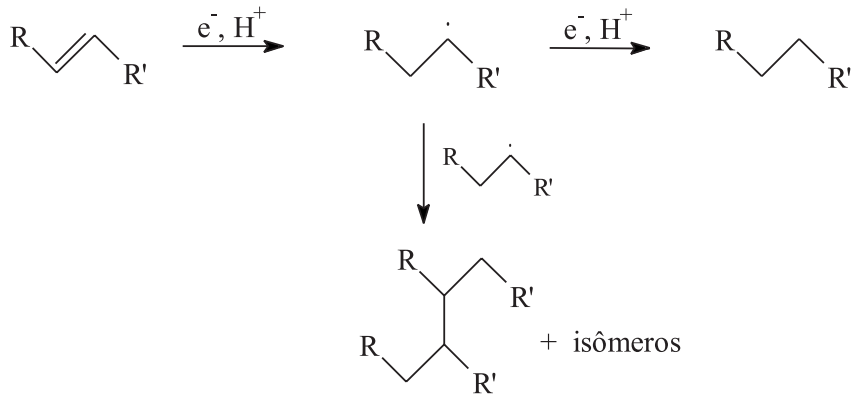

Esquema 1. Mecanismo de redução direta de olefinas via eletroquímica

A competição entre HEC, REH e DE-HE (Esquema 2) exemplifica a complexidade que um sistema eletroquímico pode atingir, caso o controle de alguns parâmetros não seja levado em consideração.

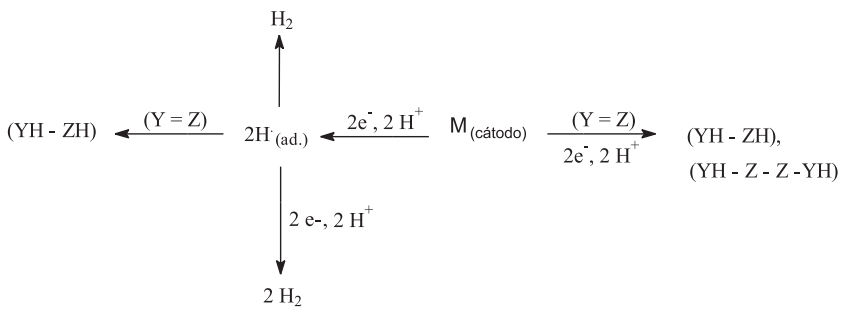

Esquema 2. Competição entre os mecanismos de redução eletroquímica direta e indireta de compostos insaturados

A eficiência eletroquímica da HEC, ou seja, carga passada para geração de $\mathrm{H} \cdot$ vs. produto de hidrogenação, é determinada pela competição entre a reação de hidrogenação de um substrato, reação de evolução de hidrogênio molecular e, em alguns casos, a redução di- reta do substrato. A velocidade relativa destes processos é afetada por alguns fatores: atividade do hidrogênio quimicamente adsorvido, densidade de corrente aplicada ao sistema, presença de qualquer molécula no sistema (solvente, eletrólito de suporte, surfactantes, etc. $)^{20}$, ou mesmo adsorção do reagente na superfície do cátodo (catalisador) $^{8,21}$.

Dois artigos de revisão devotados à HEC estão disponíveis após um século da descoberta do processo eletroquímico para a hidrogenação de substratos orgânicos ${ }^{4}$. A revisão escrita por Moutet $^{3}$ discute os importantes avanços obtidos na área, com grande ênfase na área de eletrodos modificados. A revisão relatada por $\mathrm{Beck}^{5}$, um pouco mais antiga (1979), tem um maior enfoque tecnológico e aplicativo relatando os três possíveis processos descritos acima: HEC, HC e HE. Neste artigo de divulgação temos por objetivo reportar os avanços obtidos pela técnica de HEC nos últimos 10 anos, que podem tornar o seu uso mais corriqueiro num laboratório de síntese orgânica, ou mesmo industrialmente.

\section{MATERIAIS DE ELETRODO}

O estudo da HEC pode ser enfocado de duas maneiras: estudo do material do eletrodo, que deve gerar hidrogênio radicalar, podendo funcionar ao mesmo tempo como um bom catalisador, e o estudo de diferentes classes de substratos a serem hidrogenadas. Inicialmente, cátodos compostos por metais nobres $(\mathrm{Pt}, \mathrm{Pd}, \mathrm{Rh})^{21-26}$ foram o principal objeto de trabalho, devido ao favorecimento termodinâmico $^{1}$, tanto para geração eletroquímica de hidrogênio como para o processo catalítico de hidrogenação.

O comportamento de eletrodos de platina platinizada $(\mathrm{Pt} / \mathrm{Pt})$ foi estudado por Lamy-Pitara et $a .^{27}$, onde pela primeira vez foi demonstrada a possibilidade de hidrogenação seletiva utilizando o potencial do eletrodo como uma variável para o controle da atividade (a) do catalisador (cátodo). Ácido maleico (AM), ácido metilmaleico (AMM) e dimetilmaleico (ADM) foram estudados e observou-se que a velocidade de hidrogenação variava de acordo com o número de substituintes presentes na molécula, portanto:

$\mathrm{a}_{\mathrm{AM}}>\mathrm{a}_{\mathrm{AMM}}>\mathrm{a}_{\mathrm{ADM}}$

sendo possível a hidrogenação seletiva através do controle do potencial aplicado durante a reação de hidrogenação eletrocatalítica. Quanto menor o potencial, menor a quantidade de hidrogênio atômico gerado, favorecendo a hidrogenação do substrato mais reativo.

Em um segundo trabalho os mesmos autore ${ }^{26}$ estudaram o efeito do eletrólito de suporte sobre a atividade de hidrogenação do ácido maleico, utilizando como catalisador a platina platinizada. Foi observado que o íon $\mathrm{ClO}_{4}^{-}$não interfere no processo de hidrogenação, enquanto os íons $\mathrm{HSO}_{4}^{-} \mathrm{e} \mathrm{Cl}$ interferem no processo através da forte adsorção na superfície do catalisador, na ordem de densidade de corrente $(j): j \mathrm{ClO}_{4}^{-}>j \mathrm{HSO}_{4}^{-}>j \mathrm{Cl}^{-}$.

$\mathrm{O}$ estudo de depósito de Pt sobre carbono também foi realizado com o objetivo de investigar a eficiência eletroquímica em reações de HEC vs. recobrimento do eletrodo com Pt. Foi observado que o eletrodo com depósito entre 2 e $30 \%$ não altera a eficiência eletroquímica $(\sim 70 \%)$ da HEC, no entanto eletrodos com recobrimento acima de $60 \%$ apresentaram atividade inferior a $20 \%$, demonstrando que a eficiência eletroquímica no processo de HEC do fenol depende da porcentagem de Pt no catalisador 22,23 .

Eletrodos de Pt, Pd, Ru e Rh ( $2 \%$ em C) foram testados na HEC do fenol, gerando uma mistura de cicloexanol/cicloexanona (Esquema 3), sendo que o eletrodo de Pt se mostrou mais ativo, apresentando uma eficiência eletroquímica (EE) de $72 \%{ }^{23}$. A ordem de atividade observada foi: $\mathrm{Pt}>\mathrm{Rh}>\mathrm{Pd}>\mathrm{Ru}$. 


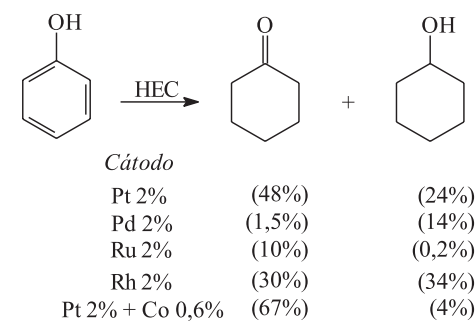

Esquema 3. Hidrogenação eletrocatalítica do fenol.

Em outro trabalho, descrito por Miller e Christensen ${ }^{21}$, também foi observada a HEC de aromáticos em condições normais de temperatura e pressão, utilizando eletrodos de Pt e Rh depositados sobre carbono (Tabela 1), onde pode-se observar claramente que o cátodo de Rh depositado sobre carbono apresentou melhores resultados como catalisador na HEC de aromáticos, com valores de até $92 \%$ de rendimento.

Tabela 1. HEC de compostos aromáticos em solução de $\mathrm{H}_{2} \mathrm{SO}_{4} 0,2 \mathrm{M}$, utilizando célula em U e Pt como ânodo ${ }^{2}$

\begin{tabular}{lccccc}
\hline Reagente & Cátodo & $\mathrm{i}(\mathrm{A})$ & $\mathrm{F}$ & \multicolumn{2}{c}{ rendimentos (\%) } \\
$\mathrm{C}_{6} \mathrm{H}_{5}-\mathrm{X} ; \mathrm{X}=$ & & & & $\mathrm{C}_{6} \mathrm{H}_{11} \mathrm{X}$ & $\mathrm{C}_{6} \mathrm{H}_{5} \mathrm{X}$ \\
\hline $\mathrm{OH}$ & $\mathrm{Pt} / \mathrm{C}$ & 0,8 & 12 & 54 & - \\
$\mathrm{OH}$ & $\mathrm{Rh} / \mathrm{C}$ & 0,8 & 7,2 & 92 & - \\
$\mathrm{NH}_{2}$ & $\mathrm{Rh} / \mathrm{C}$ & 0,8 & 12 & 73 & - \\
$\left(\mathrm{CH}_{3}\right)_{2} \mathrm{CH}$ & $\mathrm{Rh} / \mathrm{C}$ & 0,2 & 8.4 & 56 & 23 \\
$\left(\mathrm{CH}_{3}\right)_{3} \mathrm{C}$ & $\mathrm{Rh} / \mathrm{C}$ & 0,2 & 10 & 33 & 51 \\
$\mathrm{OCH}_{3}$ & $\mathrm{Rh} / \mathrm{C}$ & 0,8 & 6 & 54 & 5 \\
$\mathrm{OCH}_{3}$ & $\mathrm{Rh} / \mathrm{C}$ & 0,8 & 12 & 50 & - \\
1-metilnaftaleno & $\mathrm{Rh} / \mathrm{C}$ & 0,4 & 9 & - & 95 \\
\hline
\end{tabular}

Casadei e Pletcher ${ }^{20}$ estudaram a influência das condições experimentais na HEC para moléculas orgânicas usando diferentes tipos de materiais de cátodo: Pt, Pd, Rh e Ni de Raney depositado sobre diferentes materiais ( $\mathrm{Pt}$, grafite e poço de mercúrio). O meio reacional escolhido dentre outros para realizar as reações de HEC foi $\mathrm{MeOH} /$ $\mathrm{MeO}^{-} \mathrm{Na}^{+}(1 \mathrm{M})$. A Tabela 2 apresenta os resultados obtidos nas reações de HEC da acetofenona, utilizando $\mathrm{Pt} / \mathrm{Pt}$ como material do cátodo (catalisador). Substratos como estireno e cianobenzeno, que não apresentaram reatividade nestas condições experimentais, puderam ser hidrogenados utilizando níquel de Raney e Pd/C.

Pletcher et al. ${ }^{28,29}$ também estudaram a HEC de moléculas orgânicas utilizando eletrodo de paládio depositado na superfície de níquel e o mecanismo de geração de $\mathrm{M}-\mathrm{H}$ a partir de diferentes fontes de prótons como, por exemplo, ácido acético e metanol.

\section{NÍQUEL E COBRE COMO CATALISADORES}

Em trabalho similar ao descrito acima, Pletcher et al..$^{30,31}$ investi- garam a eletrodeposição de catalisador in situ para a hidrogenação eletrocatalítica. O processo envolveu um sal de níquel dissolvido no eletrólito que, durante o processo de HEC, resulta na deposição de Ni na superfície do eletrodo (também de Ni), permitindo a renovação constante da superfície do catalisador. Embora a idéia seja interessante, o catalisador mostrou-se mais ativo no sentido da geração de $\mathrm{H}_{2}(\mathrm{REH})$, e pouco ativo do ponto de vista da HEC.

Rubinskaya et al. ${ }^{32-36}$ estudaram a influência da composição do solvente, do $\mathrm{pH}$ no meio reacional e do tipo de eletrodo $(\mathrm{Cu}$ e $\mathrm{Ni})$ para a HEC do citral e observaram que a natureza do solvente pode influenciar fortemente o rendimento dos produtos desta reação (Esquema 4 e Tabela 3).

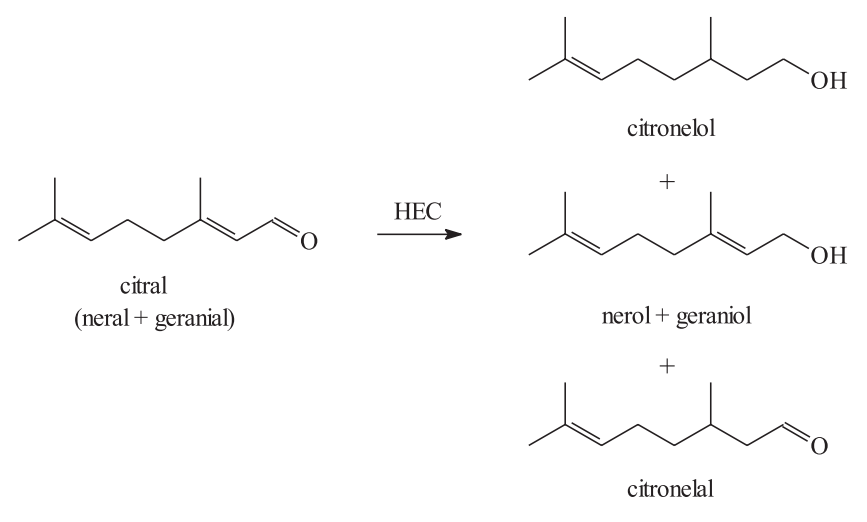

Esquema 4. Hidrogenação eletrocatalítica do citral

A proporção de DMF adicionado, assim como a utilização de outros solventes, promove uma modificação na proporção dos vários produtos de hidrogenação, além de influenciar na atividade do catalisador, diminuindo a eficiência do mesmo ${ }^{34}$. A adição de um ácido fraco ao sistema também pode ajudar na obtenção de melhores rendimentos dos produtos de hidrogenação: citronelol, geraniol e nerol $^{35}$.

Outro parâmetro investigado foi a utilização de níquel no lugar de cobre como catalisador. Vários depósitos de níquel sobre níquel foram testados e observou-se que os rendimentos da reação de HEC, os quais podem alcançar $95 \%$ do produto de hidrogenação (citronelol), variam bastante em função do método de deposição empre$\operatorname{gado}^{36}$.

Eletrodos de níquel também têm sido estudados objetivando sua aplicação na HEC. Dentre eles podemos citar eletrodos de níquel depositados na superfície de alguns materiais como Ni, Fe e grafite, além do próprio níquel de Raney. A atividade catalítica do níquel pode variar de acordo com a sua preparação. A praticidade e economia aliadas ao alto poder catalítico do níquel mostram uma tendência no sentido do desenvolvimento deste tipo de catalisador.

Eletrodos compostos por níquel de Raney foram utilizados na HEC de alguns compostos poliaromáticos (fenantreno, antraceno e naftaleno $)^{7}$, como também do benzeno, anilina e nitrobenzeno ${ }^{6}$. Neste

Tabela 2. Influência das condições experimentais na hidrogenação da acetofenona utilizando Pt/Pt como cátodo em metanol, com densidade de corrente de $50 \mathrm{~mA} \mathrm{~cm}^{-2} 20$

\begin{tabular}{lccc}
\hline Condições Experimentais & $\mathrm{C}_{6} \mathrm{H}_{5} \mathrm{C}_{2} \mathrm{H}_{5}(\%)$ & $\mathrm{C}_{6} \mathrm{H}_{5} \mathrm{CH}(\mathrm{OH}) \mathrm{CH}_{3}{ }^{*}(\%)$ & Reagente recuperado (\%) \\
\hline $\left.\mathrm{CH}_{3} \mathrm{OH} / \mathrm{CH}_{3} \mathrm{O}^{+} \mathrm{Na}^{+}(1 \mathrm{~mol} \mathrm{dm})^{-3}\right)$ & - & 85 & 12 \\
$90 \% \mathrm{CH}_{3} \mathrm{OH} / 10 \% \mathrm{H}_{2} \mathrm{O} / \mathrm{NaOH}\left(1 \mathrm{~mol} \mathrm{dm}^{-3}\right)$ & - & 82 & 12 \\
$70 \% \mathrm{CH} / \mathrm{OH} / 30 \% \mathrm{H}_{2} \mathrm{O} / \mathrm{NaOH}\left(1 \mathrm{~mol} \mathrm{dm}^{-3}\right)$ & - & 72 & 20 \\
$\mathrm{CH}_{3} \mathrm{OH} / 5 \% \mathrm{H}_{2} \mathrm{SO}_{4}$ & 30 & 36 & 29 \\
\hline
\end{tabular}

*Análises após 2 F. Quantificado como \% inicial de acetofenona. 
Tabela 3. Rendimento dos produtos da HEC do citral, em função da composição do solvente. [citral] $=0,024$ mol L ${ }^{-1},[\mathrm{AcOH}]=0.25 \mathrm{~mol} \mathrm{~L}{ }^{-1} 34$

\begin{tabular}{lcccc}
\hline $\mathrm{H}_{2} \mathrm{O}:$ Solvente $(\%)^{*}$ & Reagente Recuperado (\%) & citronelol & $\begin{array}{c}\text { Produtos (\%) } \\
\text { nerol + geraniol }\end{array}$ & citronelal \\
\hline DMF $(40 \%)$ & - & 74 & 16 & - \\
DMF (40\%) + MeOH (5\%) & - & 35 & 23 & 10 \\
DMF (40\%)+ EtOH (10\%) & - & 38 & 19 & - \\
DMF (75\%)** & 13 & 9 & 13 & 28 \\
EtOH (40\%) & 15 & 6 & 39 & 14 \\
iso-PrOH (50\%)** & 23 & - & 15 & 2 \\
EtOH (40\%) + DMF (10\%) & 33 & - & 28 & 14 \\
\hline
\end{tabular}

$* \mathrm{v} / \mathrm{v}$. **Presença de $[\mathrm{AcOH}]=0,5 \mathrm{~mol} \mathrm{~L}^{-1}$.

caso observou-se a influência do eletrólito de suporte (sais de amônio) nos rendimentos das reações de HEC, que ocorreram em temperaturas diferentes da do ambiente $\left(50\right.$ a $\left.110{ }^{\circ} \mathrm{C}\right)$.

Menini et al. ${ }^{9}$ verificaram que a HEC sob corrente controlada, pressão média (3 a $41 \mathrm{~atm})$ de um gás inerte $\left(\mathrm{N}_{2}\right)$, temperaturas de 81 a 110 ${ }^{\circ} \mathrm{C}$ e níquel de Raney como cátodo, levaram a rendimentos da ordem de 99\% para a hidrogenação do fenantreno, gerando os dois octahidrofenantrenos $(91 \%)$ e produtos di-(3\%) e tetra-hidrogenados $(5 \%)$, com uma eficiência eletroquímica de cerca de 94\% (Esquema 5).

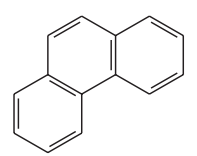

fenantreno

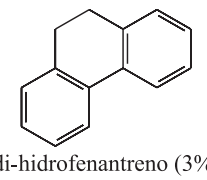

tetra-hidrofenantreno $(5 \%)$

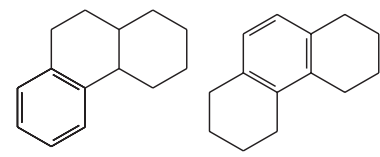

octa-hidrofenantreno $(91 \%)$
Esquema 5. Hidrogenação eletrocatalítica do fenantreno

Chambrion et al. ${ }^{8}$ realizaram a HEC de alguns diterpenos, como limoneno e carvona, gerando os respectivos produtos de hidrogenação (Esquema 6) em meio micelar emulsificado e de soluções hidroorgânicas (água:metanol), utilizando eletrodos de níquel de Raney. Foi observado que a presença de um surfactante pode aumentar a adsorção do substrato na superfície do eletrodo de duas maneiras: (i) aumentando a solubilidade do substrato no meio aquoso ou (ii) pela formação de uma camada de surfactante adsorvida na superfície do cátodo, na qual o substrato pode ser incorporado. Vários surfactantes foram testados: brometo de cetiltrimetilamônio (CTAB, catiônico), dodecilssulfato de sódio (SDS, aniônico) e Brij-35 (neutro). Os melhores resultados obtidos para a HEC, na presença de surfactantes, foram verificados em soluções micelares de surfactantes catiônicos, por exemplo, na HEC do limoneno em meio micelar de CTAB foi obtido $57 \%$ de $p$-mentano.

A utilização de níquel de Raney e outros tipos de eletrodos de níquel também tem sido aplicada à HEC seletiva de olefinas conjugadas. Bryan e Grimshaw ${ }^{37}$ descreveram que a hidrogenação seletiva da dupla ligação carbono-carbono conjugada presente em 4fenilbuten-2-onas (Esquema 7), utilizando cátodo de $\mathrm{Ni}$ sobre $\mathrm{Cu}$, dependia criticamente do método de eletrodeposição usado na preparação da superfície do eletrodo de níquel. Dentre as três metodologias de preparação do eletrodo de níquel (níquel cinza, níquel de Ishiwata e negro de níquel), o eletrodo de negro de níquel foi o

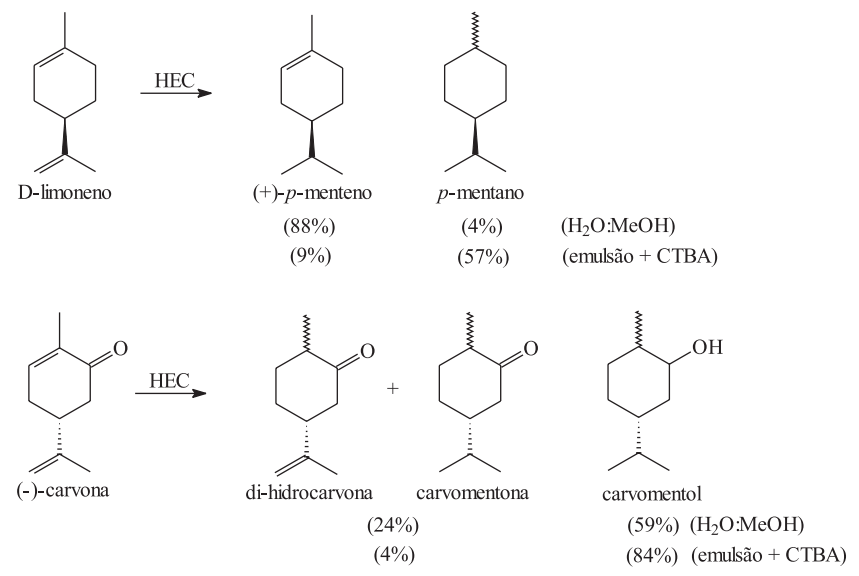

Esquema 6. Hidrogenação eletrocatalítica do limoneno e carvona

que mostrou eficiência eletroquímica mais elevada (63-68\%), dando uma mistura de alcanona e o álcool secundário correspondente. Finalmente, este processo mostrou ser uma rota alternativa para a conversão de 4-fenilbuten-2-onas em 4-fenilbutanonas, no qual não há necessidade de fonte de hidrogênio gasoso.

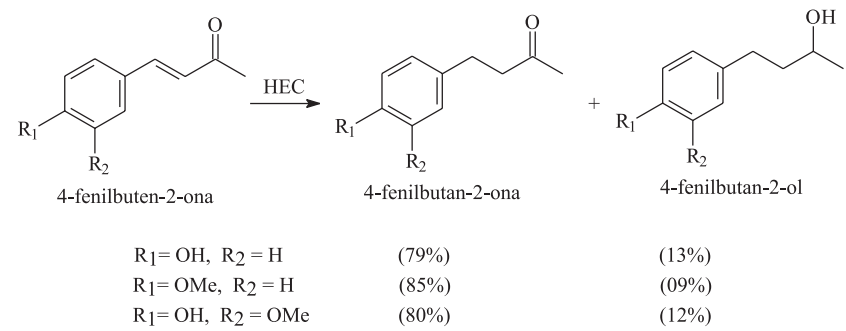

Esquema 7. Hidrogenação eletrocatalítica de 4-fenilbuten-2-ona

Mahdavi et $a l .{ }^{38}$ realizaram um estudo da HEC com olefinas conjugadas (2-cicloexen-1-ona, acetilcicloexeno, 3-isopropilideno6-metil-2-cicloexen-1-ona, etc.), o qual demonstrou que o eletrodo de níquel fractal apresentou alta seletividade de hidrogenação (99\%) com relação a duplas conjugadas em $\alpha, \beta$ à carbonila, quando comparados aos dados obtidos utilizando-se eletrodos de boreto de níquel e níquel de Raney. No entanto, Dabo et al. ${ }^{39}$ também realizaram um estudo de HEC com 2-cicloexen-1-ona para obtenção da cicloexanona e verificaram que ao utilizar eletrodos de $\mathrm{Ni}$ e $\mathrm{Cu}$ como catalisadores, a seletividade foi muito maior quando comparada ao uso do eletrodo de níquel fractal (Esquema 8). 


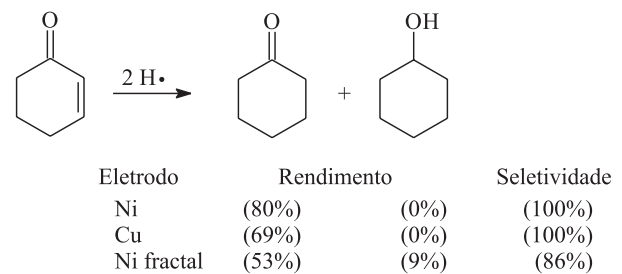

Esquema 8. Hidrogenação eletrocatalítica da 2-cicloexen-1-ona

Yusem e Pintauro ${ }^{40}$ descreveram a hidrogenação do óleo de soja via HEC usando o sistema de solvente água/t-butanol (1:1) e ptoluenossulfonato de tetrabutilamônio $(1,0 \mathrm{M})$ como eletrólito de suporte, à temperatura de $70{ }^{\circ} \mathrm{C}$ e utilizando um eletrodo de níquel de Raney como catalisador. O grau de saturação de ácidos graxos, usado para quantificar os produtos de hidrogenação, foi similar aos resultados obtidos pela hidrogenação catalítica tradicional (HC) em altas temperaturas, mas se verificou que, pela técnica eletrocatalítica, houve um aumento significativo de produto de hidrogenação total, determinado através da quantificação do ácido esteárico presente na amostra (0,5\% para $\mathrm{HC} / 1,0-4,5 \%$ para $\mathrm{HEC})$.

\section{MATERIAIS ALTERNATIVOS DE ELETRODO}

Alguns materiais alternativos também têm sido estudados na HEC. Van Druten et al. ${ }^{41}$ relataram um novo processo de hidrogenação eletrocatalítica utilizando $\mathrm{LaNi}_{5}$ como material do cátodo, solventes polares (etanol e água) como meio reacional, pressão e temperatura ambientes. Este material demonstrou ter a capacidade de armazenar hidrogênio já que, em presença de $\mathrm{H}_{2}$, gerava $\mathrm{LaNiH}_{4}$. A hidrogenação de metilvinilcetona, 1-deceno e 5-hexen-2-ona, por este método, apresentou seletividade gerando produtos que tiveram apenas as duplas ligações carbono-carbono hidrogenadas.

Navarro et al. ${ }^{19}$ descreveram um método simples e eficaz de hidrogenação de olefinas conjugadas (eletroquimicamente ativas), maleato e fumarato de dietila (este último foi utilizado como modelo), com um potencial de redução pouco negativo (vs. $\mathrm{Ag} / \mathrm{AgCl}$ ). O método descrito envolveu a utilização de uma célula eletroquímica unitária (Figura 1) com um ânodo dimensionalmente estável $\left(\mathrm{RuO}_{2} /\right.$ $\mathrm{TiO}_{2}$ ) e um cátodo, no qual foram testados diversos materiais: $\mathrm{Pt}, \mathrm{Fe}$, $\mathrm{C}, \mathrm{Ni}, \mathrm{Pb}, \mathrm{Al}, \mathrm{Cu}, \mathrm{Zn}$ e aço inox. Neste método de hidrogenação foi observado um comportamento competitivo entre a hidrogenação e a dimerização, fato este atribuído à redução da olefina conjugada e à recombinação a partir do ânion radical formado (Esquema 9). O eletrodo de ferro foi o que apresentou maior seletividade com relação à hidrogenação da olefina conjugada. As melhores condições de hidrogenação do fumarato de dietila foram observadas ao se utilizar $\mathrm{NaCl}$ como eletrólito suporte $(0,1 \mathrm{M})$, co-solvente acetonitrila, densidade de corrente $175 \mathrm{~mA} \mathrm{dm}{ }^{-2}$ e uma carga de $4 \mathrm{~F}$. Nenhuma dependência do $\mathrm{pH}$ no meio reacional foi evidenciada. $\mathrm{O}$ processo de hidrogenação neste caso ocorre tanto pelo processo catalítico como pelo processo eletroquímico (Tabela 4).

Em outro trabalho, Navarro et al. ${ }^{42}$ desenvolveram um sistema eletrocatalítico para hidrogenação de aldeídos, cetonas e olefinas conjugadas usando um ânodo de sacrifício. O sistema apresentado no Esquema 10 mostra como o ânodo de níquel pode ter dupla função no sistema: ânodo de sacrifício e ativador do material do cátodo usado como catalisador. Este processo permite a utilização de uma célula de compartimento único (Figura 1) e a constante deposição de níquel no cátodo, simultânea ao processo de HEC do substrato, promove a renovação da superfície do catalisador e impede o envenenamento do mesmo após um certo período de catálise ${ }^{43}$. Os rendimen-

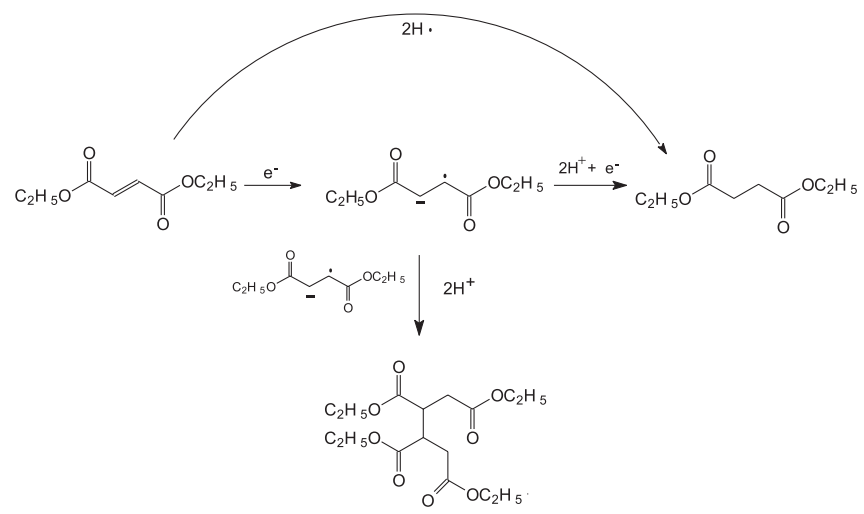

Esquema 9. Hidrogenação do fumarato de dietila utilizando Fe como cátodo

Tabela 4. HEC do fumarato de dietila em água/acetonitrila (4:1), utilizando $\mathrm{NaCl}$ 0,1 M como eletrólito de suporte ${ }^{42}$

\begin{tabular}{|c|c|c|c|c|}
\hline \multirow[b]{2}{*}{ Cátodo } & \multirow[b]{2}{*}{$\begin{array}{l}\text { Densidade } \\
\text { de Corrente } \\
\left(\mathrm{mA} \mathrm{dm}{ }^{-2}\right)\end{array}$} & \multicolumn{3}{|c|}{ Produtos $(\%)$} \\
\hline & & $\begin{array}{l}\text { Fumarato } \\
\text { de dietila }\end{array}$ & $\begin{array}{l}\text { Succinato } \\
\text { de dietila }\end{array}$ & $\begin{array}{c}\text { Dímero } \\
\text { (tetraéster) }\end{array}$ \\
\hline $\mathrm{Fe}$ & 175 & 3 & 84 & 5 \\
\hline $\mathrm{Pt}$ & 265 & 0 & 50 & 30 \\
\hline Feltro de C & 265 & 1 & 30 & 51 \\
\hline $\mathrm{Ni}$ & 265 & 17 & 8 & 18 \\
\hline $\mathrm{Pb}$ & 265 & 0 & 52 & 34 \\
\hline $\mathrm{Al}$ & 265 & 0 & 44 & 28 \\
\hline $\mathrm{Cu}$ & 265 & 0 & 40 & 35 \\
\hline $\mathrm{Zn}$ & 265 & 0 & 53 & 14 \\
\hline aço Inox & 175 & 18 & 42 & 10 \\
\hline
\end{tabular}

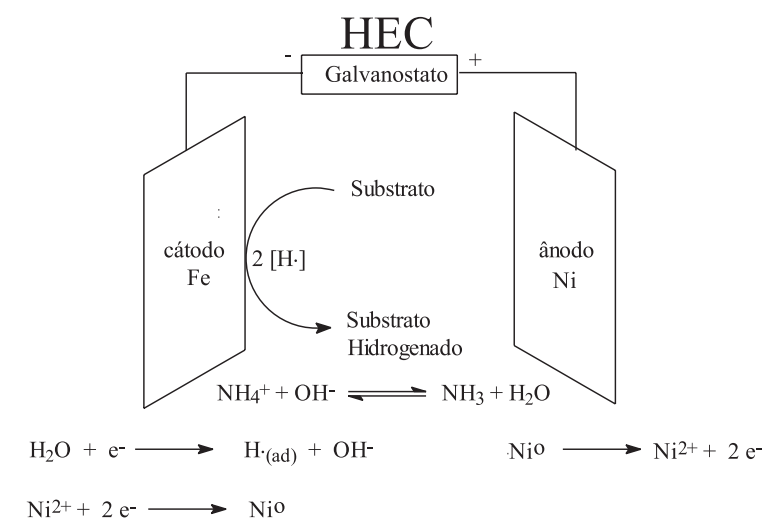

Esquema 10. Representação do sistema para HEC, utilizando ânodo de sacrifício de $\mathrm{Ni}$

tos obtidos na redução de olefinas conjugadas (2-cicloexen-1-ona, benzaldeído, acetofenona, estireno, etc.) demonstram o método de HEC, com os seguintes parâmetros: célula unitária, ânodo de sacrifício de níquel, barra de ferro como cátodo, concentração $0,1 \mathrm{M}$ de substrato em água/metanol (1:1), 0,2 M de acetato ou cloreto de amônio como eletrólito de suporte e densidade de corrente variando de 350 a $175 \mathrm{~mA} \mathrm{dm}^{-2}$. Nestas condições foi observada seletividade com relação à redução das duplas ligações, aliada a excelentes rendimentos (88-95\%) e uma boa eficiência eletroquímica $(50-80 \%)^{42}$. 


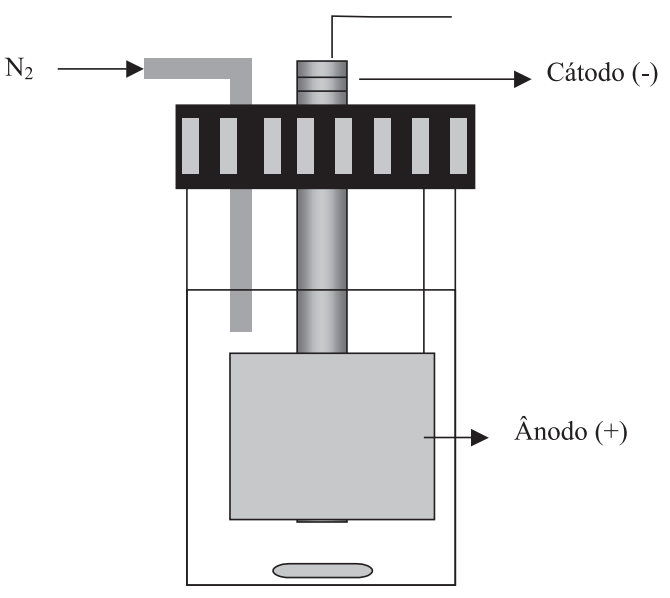

Figura 1. Célula eletroquímica de compartimento único utilizada na HEC com ânodo de sacrifício

\section{ELETRODOS MODIFICADOS POLIMÉRICOS}

Polímeros condutores podem ser utilizados para a imobilização de diversos complexos metálicos ${ }^{44,45}$ ou micropartículas metálicas ${ }^{46}$ capazes de realizar a reação de hidrogenação. A HEC pode ocorrer através da imobilização de micropartículas metálicas dispersas dentro de diversos polímeros que podem ser utilizados para preparação do eletrodo modificado, visando a geração de hidrogênio via fotoquímica ou eletroquímica: poli(ácido vinil acético $)^{47}$, poli(3-metiltiofeno ${ }^{48}$, poli(4-vinilpiridina) ${ }^{49}$, Nafion ${ }^{50}$, polianilina ${ }^{51}$, poli(mercapto-hidroquinona ou benzoquinona) $)^{52}$. Outros trabalhos relatam eletrodos modificados contendo partículas de Pt, Pd e Rh utilizando como polímero suporte poli(pirrolviologênio) $)^{53-55}$, poli(pirrol hidrogenosulfato $)^{56} \mathrm{ou}$ mesmo o uso de micropartículas de Ni dispersas em poli(pirrol alquilamônio) ${ }^{46}$. O Esquema 11 exemplifica como ocorre o processo de HEC em eletrodo modificado de polipirrol, utilizando viologênio como transferidor de elétrons dentro do eletrodo modificado ${ }^{55}$.

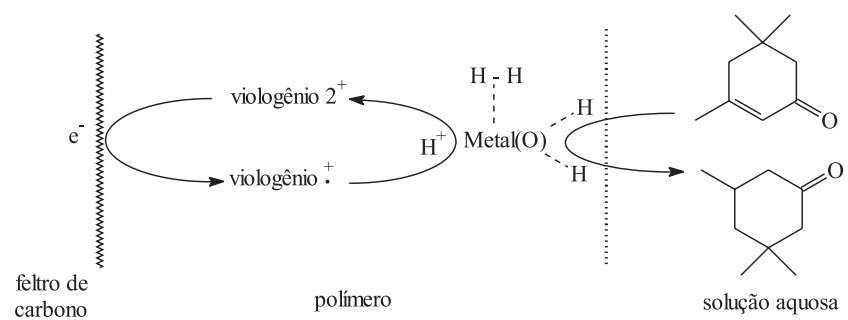

Esquema 11. Hidrogenação eletrocatalítica de uma olefina em eletrodo modificado de C/poli(pirrolviologênio)-M(O); onde $M=P d$, Pt ou $R h ; V^{2+}$ / $V^{++}$é o sistema redox dicátion 4,4'bipiridínio/ radical-cátion, ligado à matriz. polipirrólica

\section{HIDROGENAÇÃO ELETROCATALÍTICA DE OUTRAS CLASSES DE SUBSTRATOS}

A comparação entre resultados obtidos pelos métodos direto e indireto de redução mostra que existe uma maior seletividade para o processo catalítico. É o que se pode observar nos trabalhos descritos a seguir.

Marquez e Pletcher ${ }^{57}$ estudaram as condições experimentais para a redução eletroquímica direta de $o$-halonitrobenzenos às anilinas correspondentes e sugeriram que a redução ocorre em três etapas
(Esquema 12). Para compostos substituídos com hidrogênio, flúor e cloro, os rendimentos chegaram a exceder $75 \%$ (densidade de corrente de $100 \mathrm{~mA} \mathrm{~cm}^{-2}, 0,1 \mathrm{M}$ de $\mathrm{H}_{2} \mathrm{SO}_{4}$ como eletrólito de suporte e eletrodo de mercúrio), já para $o$-bromonitrobenzeno o rendimento foi baixo (42\%), devido à competição da quebra da ligação C-Br.

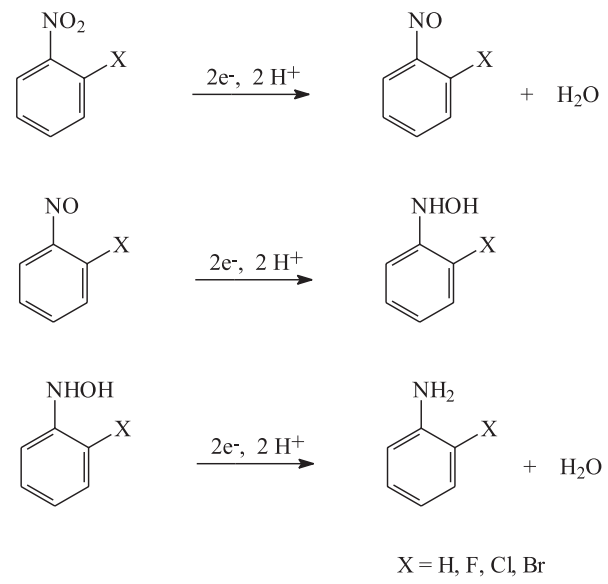

Esquema 12. Mecanismo de redução eletroquímica direta de nitrobenzenos substituídos

Cyr et $a l .{ }^{58}$ realizaram com sucesso a redução preparativa de nitrobenzeno, azoxibenzeno, azobenzeno e hidrazobenzeno pelo processo de HEC (método indireto), obtendo como produtos as respectivas anilinas (rendimento químico de $85-100 \%$ e eficiência eletroquímica de 80-100\%), em solução metanólica aquosa (neutra ou básica) utilizando eletrodos de cobre de Devarda e níquel de Raney. A explicação para estes rendimentos foi indicada pela hidrogenólise das ligações: N-O para a fenil-hidroxilamina e N-N para hidrazobenzeno desprotonado, onde baixos potenciais no cátodo são devidos aos processos de hidrogenação catalítica, ou seja, reação do substrato orgânico com hidrogênio quimicamente adsorvido, equações de 1-5:

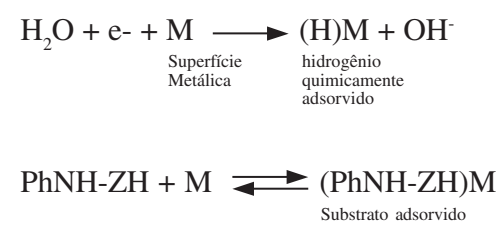

$(\mathrm{PhNH}-\mathrm{ZH}) \mathrm{M}+2(\mathrm{H}) \mathrm{M} \longrightarrow\left(\mathrm{PhNH}_{2}\right) \mathrm{M}+\left(\mathrm{ZH}_{2}\right) \mathrm{M}$

$\left(\mathrm{PhNH}_{2}\right) \mathrm{M} \longrightarrow \mathrm{PhNH}_{2}+\mathrm{M}$

$\left(\mathrm{ZH}_{2}\right) \mathrm{M} \rightleftarrows \mathrm{ZH}_{2}+\mathrm{M}$

Segundo os autores, este foi o primeiro exemplo de conversão eletroquímica eficiente de nitrobenzeno, azoxi e azobenzeno em anilina em meio neutro e básico.

Em 1993, Cleghorn e Pletcher ${ }^{28}$ publicaram um estudo sobre uma eficiente conversão (80\%), com alta seletividade do cátodo (paládio e níquel) em meio metanólico ácido, mas apenas para a redução de nitrobenzeno para anilina (87\%, eficiência eletroquímica de $92 \%)$.

\section{CONCLUSÕES E PERSPECTIVAS}

Com o desenvolvimento de novos materiais e métodos de preparação de eletrodos catalíticos, somado ao estudo das condições experimentais ideais para realização de reações de hidrogenação atra- 
vés do método eletroquímico, a literatura demonstra que os avanços recentes podem tornar a HEC uma técnica mais atrativa, quando comparada à $\mathrm{HC}$ convencional. Algumas vantagens da técnica, tais como utilização de condições brandas, ausência da necessidade de hidrogênio gasoso e o desenvolvimento de condições experimentais simplificadas (uso de água e/ou metanol como meio reacional, células eletroquímicas não divididas, eletrodos obtidos a partir de materiais ou técnicas simples, eletrodos com características de especificidade ou seletividade), poderão tornar a HEC um método de hidrogenação mais conhecido e útil na síntese orgânica.

\section{AGRADECIMENTOS}

Os autores agradecem à FINEP/CTPETRO/PETROBRAS pelo apoio financeiro.

\section{REFERÊNCIAS}

1. Mcgrath, M. P.; Sall E. D.; Tremont S. J.; Chem. Rev. 1995, 95, 381; Johnstone, R. A. W.; Wilby, A. H.; Entwistle, I. D.; Chem. Rev. 1985, 85, 129; Hoelscher, H. E.; Poynter, W. G.; Weger, E.; Chem. Rev. 1954, 54, 575; Brieger, G.; Nestrick, T. J.; Chem. Rev. 1974, 74, 567; House, H. O.; Modern Synthetic Reactions, $2^{\text {nd }}$ ed., W. A. Benjamin: Menlo Park, 1972, cap. 1.

2. Zaera, F.; Chem. Rev. 1995, 95, 2651; Esteruelas, M. A.; Ore, A.; Chem. Rev. 1998, 98, 577; Naota, T.; Takaya, H.; Murahashi, S. I.; Chem. Rev 1998, 98, 2599; Zassinovich, G.; Mestroni, G.; Gladiali, S.; Chem. Rev. 1992, 92, 1051; Harmon, R. E.; Gupta, S. K.; Brown, D. J.; Chem. Rev $1973,73,21$.

3. Moutet J.-C.; Org. Prep. Proced. Int. 1992, 24, 309; Moutet J.-C.; Actual. Chimique 1998, 63.

4. Haber, F.; Phisik. Chem. 1900, 32, 193; Fokin, S. Z.; Elektrochem. 1906, 12, 749; Haber, F.; Phisik. Chem. 1900, 32, 193.

5. Beck, F.; Int. Chem. Eng. 1979, 19, 1

6. Pintauro, P. N.; Bontha, J. R.; J. Appl. Electrochem. 1991, 21, 799.

7. Robin, D.; Comtois, M.; Martel, A.; Lemieux, R.; Cheong, A. K.; Belot, G.; Lessard, J.; Can. J. Chem. 1990, 68, 1218.

8. Chambrion, P.; Roger, L.; Lessard, J.; Béraud, V.; Mailhot, J.; Thomalla, M.; Can. J. Chem. 1995, 73, 804

9. Menini, R.; Martel, A.; Ménard, H.; Lessard, J.; Vittori, O.; Electrochim Acta 1998, 43, 1697; Mahdavi, B.; Chapuzet, J. M.; Lessard, J.; Electrochim. Acta 1993, 38, 1377.

10. Trasatti, S. Em Advances in Electrochemical Science and Engineering; Gerisher, H.; Tobias, C. W., eds.;VCH: Weinhein, 1991, vol. 2, p. 1-85.

11. Parsons, R.; Trans Faraday Soc. 1958, 54, 1053.

12. Bockris, J. O'M. Em Modern Aspects of Electrochemistry; Bockris, J. O'M.; Conway, B. E., eds.; Butterworths: London, 1954, p. 180.

13. Thomas, J. G. N.; Trans Faraday Soc. 1961, 57, 1603.

14. Conway, B. E.; Salomon, N.; Electrochim. Acta 1964, 19, 117.

15. Fedkiw, P. S.; Scott, W. D.; J. Electrochem. Soc. 1984, 139, 1058; Fedkiw, P. S.; Chao, J. C.; AIChE J. 1985, 31, 1578.

16. Popp, F. D.; Schultz, H. P.; Chem. Rev. 1962, 61, 19.

17. Nadjo, L.; Savéant, J. M.; Tessier, D.; J. Electroanal. Chem. 1975, 64, 143.

18. Chan-Shing, E. S.; Boucher, D.; Lessard, J.; Can. J. Chem. 1999, 77, 687.

19. Da Silva Jr., J. G.; Goulart, M. O. F.; Navarro, M.; Tetrahedron 1999, 55, 7405 .

20. Casadei. M. A.; Pletcher, D.; Electrochim. Acta 1988, 33, 117.
21. Miller, L. L.; Christensen, L.; J. Org. Chem. 1978, 43, 2059.

22. Amouzegar, K.; Savadogo, O.; Electrochim. Acta 1994, 39, 557.

23. Amouzegar, K.; Savadogo, O.; J. Appl. Electrochem. 1997, 27, 539

24. Amouzegar, K.; Savadogo, O.; Electrochim. Acta 1998, 43, 503.

25. Quiroz, M. A.; Córdova, F.; Lamy-Pitara, E.; Barbier, J.; Electrochim. Acta 2000, 45, 4291.

26. Lamy-Pitara, E.; El Mouahid, S.; Barbier, J.; Electrochim. Acta 2000, 45, 4299.

27. Lamy-Pitara, E.; Belegridi, I.; Barbier, J.; Catal. Today 1995, 24, 151.

28. Cleghorn, S. J. C.; Pletcher, D.; Electrochim. Acta 1993, 38, 425.

29. Cleghorn, S. J. C.; Lain, M. J.; Pletcher, D.; Electrochim. Acta 1993, 38, 2683.

30. Lain, M. J.; Pletcher, D.; Electrochim. Acta 1987, 32, 99.

31. Lain, M. J.; Pletcher, D.; Electrochim. Acta 1987, 32, 109.

32. Rubinskaya, T. Y.; Korotayeva, L. M.; Gultyai, V. P.; Russ. Chem. Bull. 1993, 42, 1830. (CA 122:276508s).

33. Korotayeva, L. M.; Rubinskaya, T. Y.; Gultyai, V. P.; Russ. Chem. Bull. 1993, 42, 1835. (CA 122:276509t)

34. Rubinskaya, T. Y.; Korotayeva, L. M.; Gultyai, V. P.; Russ. Chem. Bull. 1996, 45, 74. (CA 125:11142y).

35. Korotayeva, L. M.; Rubinskaya, T. Y.; Gultyai, V. P.; Russ. Chem. Bull. 1997, 46, 459. (CA 127:226487s).

36. Korotayeva, L. M.; Rubinskaya, T. Y.; Gultyai, V. P.; Russ. Chem. Bull. 1998, 47, 1487. (CA 129:295172p).

37. Bryan A.; Grimshaw, J.; Eletrochim. Acta 1997, 42, 2101.

38. Mahdavi, B.; Chambrion, P.; Binette, J.; Martel, E.; Lessard, J.; Can. J. Chem. 1995, 73, 846

39. Dabo, P.; Mahdavi, B.; Ménard, H.; Lessard, J.; Electrochim. Acta 1997, 42, 1457.

40. Yusem, G. J.; Pintauro, P. N.; J. Am. Oil Chem. Soc. 1992, 69, 399.

41. Van Druten, G. M. R.; Labbé, E.; Paul-Boncour, V.; Périchon, J.; PercheronGuégan, A.; J. Electroanal. Chem. 2000, 487, 31.

42. Santana, D. S.; Melo, G. O.; Lima, M. V. F.; Daniel, J. R. R.; Areias, M. C. C.; Navarro, M.; J. Electroanal. Chem. 2004, no prelo; Santana, D. S.; Lima, M. V. F.; Daniel, J. R. R.; Navarro, M.; Tetrahedron Lett. 2003, 44, 4725 .

43. Navarro, D. M. A. F.; Navarro M.; J. Chem. Educ., no prelo.

44. Bettega, H. C.-Y.; Moutet, J.-C.; Tyngry, S.; J. Electroanal. Chem. 1995, 391,51

45. Hamar-Thimbault, S.; Moutet, J.-C.; Tyngry, S.; J. Organomet. Chem. 1997, 532,31 .

46. Zouaoui, A.; Stéphan, O.; Ourari, A.; Moutet, J.-C.; Electrochim. Acta 2000, $46,49$.

47. Kao, W. H.; Kuwana, T.; J. Am. Chem. Soc. 1984, 106, 473

48. Tourillon, G.; Garnier, F.; J. Phys. Chem. 1984, 88, 5281

49. Bartak, D. E.; Kazee, B.; Shimazy, K.; Kuwana, T.; Anal. Chem. 1986, 58, 2756; Kost, K.; Bartak, D. E.; Kazee, B.; Kuwana, T.; Anal. Chem. 1990, $62,151$.

50. Itaya, K.; Takahashi, H.; Uchida, I.; J. Electroanal. Chem. 1986, 208, 373.

51. Kost, K.; Bartak, D. E.; Kazee, B.; Kuwana, T.; Anal. Chem. 1986, 60, 2379.

52. Arai, G.; Matsumoto, K.; Murofushi, T.; Yasumori, Y.; Bull. Chem. Soc. Jpn. 1990, 63, 121.

53. Coche, L.; Moutet, J.-C.; J. Am. Chem. Soc. 1987, 109, 6887.

54. Coche, L.; Ehui, B.; Limosin, D.; Moutet, J.-C.; J. Org. Chem. 1990, 55 , 5905.

55. Deronzier, A.; Moutet, J.-C.; Acc. Chem. Res. 1989, 22, 249.

56. Lofrano, R. C. Z.; Queiroz, J. V.; Romero, J. R.; J. Mol. Catal. A: Chem. 2001, 174, 231.

57. Marquez, J.; Pletcher, D.; Electrochim. Acta 1981, 26, 1751.

58. Cyr, A.; Hout, P.; Belot, G.; Lessard, J.; Electrochim. Acta 1990, 35, 147. 